\title{
ARTIFICIAL INTELLIGENCE IN 5G TECHNOLOGY: OVERVIEW OF SYSTEM MODELS
}

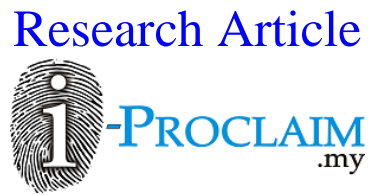

\section{Md. Mostafijur Rahman ${ }^{1}$, Mani Manavalan', Taposh Kumar Neogy ${ }^{3}$}

Asia Pac. j. energy environ.

${ }^{1}$ Lecturer, Department of Business Administration, First Capital University of Bangladesh, Alamdanga Road, Chuadanga, BANGLADESH

${ }^{2} \mathrm{Sr}$. Architect, Capgemini America, Inc., UNITED STATES

${ }^{3}$ Ph.D., Department of Accounting and Information Systems (AIS), University of Rajshahi, Rajshahi - 6205, BANGLADESH

*Email for Correspondence: mani.manavalan@ capgemini.com

\begin{abstract}
The occurrence of various devices that are interlinked to provide advanced connectivity throughout the systems revolves around the formation of $5 \mathrm{G}$ systems. Artificial Intelligence plays a fundamental role in the $5 \mathrm{G}$ networks. The popularity and integration of $5 \mathrm{G}$ have emerged through advanced cellular networks and many other technologies. This innovative and speedy network has built strong connections in recent years, its conduct in business, personal work, or daily life. Artificial Intelligence and edge computing devices have optimized internet usages in everyday life. The growth of $5 \mathrm{G}$ networks is effective in the $\mathrm{AI} / \mathrm{ML}$ algorithms due to its low latency and high bandwidth, which also performs real-time analysis, reasoning, and optimization. The $5 \mathrm{G}$ era has fundamental features that are highlighted among the revolutionary techniques which are most commonly used by cellular device networks, such as the resource management of radio, mobility management, and service management, and so on. This work also integrates the selection of spectrum and access the spectrum which AI-based interface to accomplish demands of 5G. The strategies which are introduced are Fractional Knapsack Greedy-based strategy and Language Hyperplane approach which becomes the basis of subsequently utilized by strategies of Artificial Intelligence for purpose of the selection of spectrum and the right allocation of spectrum for IoT-enabled sensor networks.
\end{abstract}

Key words

Spectrum Selection, IoT, Artificial Intelligence, AI-based Interface, 5G Technology

This article is is licensed under a Creative Commons Attribution-NonCommercial 4.0 International License.

Attribution-Non Commercial (CC BY-NC) license lets others remix, tweak, and build upon work non-commercially, and although the new works must also

acknowledge \& be non-commercial.

\section{INTRODUCTION}

The Fifth Generation (5G) network which is based on the Internet of Things (IoT) stimulates greater capacity data speeds with low latency. The fundamental use of IoT network is to provide to heterogeneous wireless users or machine devices (Bynagari, 2019; Manavalan, 2020). The 5G networks are at high demand today as they are expected to drive ultra- highspeed data transmission which is equivalent to 1000 times the speed of current LTE networks, with a higher battery life. To handle packets of data, Artificial Intelligence based approaches are used in resource management amongst the devices that are used in 5G networks. They possess the capacity to satisfy different requirements of various services for a fully connected smart society which includes services including the enhanced mobile broadband (eMBB), massive machine type communication (mMTC), and ultra-reliable and low-latency communication (URLCC). With all the specifications, 5G networks are utilized for on-demand constructions of network slices by using software defined networking (SDN) technologies. An innovative network slice which is controlled by software is also called as network softwarization. With the growing era there are various categories of wireless technologies which access the rise in fulfilling the spectrum. During the process, it improves all the channels using Fractional Knapsack. The delay in spectrum access is done by introducing Language Hyperplane Optimum Channel (spectrum) Access. It does not just minimize time lag in spectrum has the accessibility of spectrum but also boasts the spectrum accessibility and positive results. 


\section{LITERATURE REVIEW}

Cognitive radio network (CRN), and detailed examination of occupied spectrum was presented in (Azmat et al., 2016). Both supervised and unsupervised techniques were examined to determine which had the highest categorization accuracy. The interference rate, on the other hand, was not targeted, creating a significant technical obstacle to spectrum allocation. Giving in-depth Learning model for allocation of optimized spectrum which was built in order to address this problem with a Learning model to gain optimal spectrum allocation which was made in ( $\mathrm{Li}$ and Guo, 2020) that not only gave higher performance but also showed quick convergence. The total rate of D2D links is improved by using a learning algorithm to lower the likelihood of cellular links going down. However, it was unable to select resource blocks automatically (RB). Spectrum utilization becomes crucial due to a scarcity of spectrum. Artificial intelligence (AI) was first introduced in (Lin et al., 2019) to increase the credibility of spectrum utilization. The issue of spectrum scarcity has not been resolved. Another model of spectrum which is used for 5G networks was presented in detailed information to increase link rate and achieve good targets in ( $\mathrm{Li}$ and Guo, 2019). However, it was unable to complete the categorization task. Another AI-enabled 5G network was presented in for addressing multiple parts of the 5G network based on configuration and detection in (Yao et al., 2019). The spectrum shared was performed in (Huang et al., 2017) via AI methods to attain the quality of services provided (QoS) The spectrum precision, on the other hand, was not targeted. In the year 2000, work was started on employing Strategy Nash Equilibrium (SNE) to address spectrum underutilization. In (Huang et al., 2017) power AI algorithms were used to dynamically share the spectrum in order to achieve a tolerable quality of service (QoS). The spectrum precision, on the other hand, was not targeted. The use of Strategy Nash Equilibrium (SNE) to reduce spectrum underutilization was designed in (Fan et al., 2018; Ahmed \& Ganapathy, 2021; Sharma et al., 2021) to optimize latency. However, the incomplete information was not taken in action.

\section{The INTELLIGENCE IN 5G NetWORKS}

The Artificial Intelligence include the mechanism and science of developing technologies which is as diligent as humans, which is also being introduced in the communication networks (Wang et al., 2015). Artificial Intelligence is vast and it has been classified into two sections the basic or the first level determines that machine provides various options and responds differently to the environment. The second level of AI enables the machine to work completely to its full capacity to optimize such as sense, predict, mine and reason with the surroundings. The quality of machine is determined when it is able to handle any task even when it faces tough or strange scenarios to cellular networks (Amin \& Manavalan, 2017; Bynagari \& Ahmed, 2021; Bynagari \& Amin, 2019; Fadziso \& Manavalan, 2017); Manavalan, 2019a; Manavalan \& Chisty, 2019). In this step we are going to focus on how technologies of candidate provide intelligence preliminary which is the initial stage and transforming networks of cellular technology from network-centric to user-centric and information-centric with a proper SE and EE versions.

\section{Radio source management}

4G cellular networks accessibilities dependent on the on orthogonal division of frequency multiplexing (OFDM) for handling signals and works as a base associated on accessible areas. The frequency-division duplex (FDD) and timedivision duplex (TDD) formats, offers great usage by the OFDM and TDD 4G cellular networks have structure that shares similarity of frame in which they group a static number of symbols and subcarriers into one resource block (RB). It increases the satisfaction level of subcarrier in OFDM. It carries multiple set of information which can be executed individually by the receiver with least cost required for computation. For this network, 5G cellular networks always target to bring latest forms, waves and provide interfaces with softer air. Specifically, the filter-bank multi-carrier (FBMC) and unified-filter multi-carrier (UFMC) are famous candidates that give more flexibility and reliability in structured frame and news waveforms in the era of 5G. FBMC and UFMC both add filters to look for the leakage across subcarriers which makes it negligible to strictly balance across RBs. 5G cellular networks also provide various interface solutions which contains various RBs, includes multiple access schemes, TTI (transmission time interval) parameters, waveforms, and duplex mode, pilot signals, etc., can be well defined (I et al., 2016). In the given in Fig. 1a, eMBB having bandwidth which is large and symbol can provide a higher rate, whereas in URLLC, it provides a smaller TTI which can be used to reduce the latency level where It can respond rightly.

The cellular networks of $5 \mathrm{G}$ provide non-orthogonal multiple access (NoMA) techniques which includes coding multiple access, which is alike and the progression from OFDM to FBMC/UFMC (SCMA). SIC receivers (or even more computationally-exhaustive closest receivers) are used to decode the received information in such NoMA schemes, which gives overlapped information from two transmitters in the radio resource which is similar to it. Another benefit of NoMA is that it provides free transmission in the uplink (UL) if the UE identification and the grant-free UL transmission are mapped together. SIC receivers gives an overlapped information from two UEs that uses same resources instead wait for resource allocation commands like the one in $4 \mathrm{G}$ cellular networks. It grants transmission in 
UL which could eliminate the laborious signalling methods and save the latency power for tiny packets at a negligible performance loss, notwithstanding the reliability advantage of granted transmission (see Fig. 1b). In comparison, 5G cellular networks will eventually offer one alternative choice that is well suited to mMTC service.

\section{Mobility Management}

There are two states in 4G cellular networks for managing involves spreading the location of UE's to the utmost level which includes Evolved packet system (EPS). To ensure session continuity and information reachability, all the connections to EPS mobility management (EMM) and UEs should respond to their whereabouts on a regular basis. Treating all UEs the same is unquestionably resource-intensive. Instead, some UEs are in fixed places (e.g., UEs for mMTC metering services) should only emphasis at the beginning of the network connection. All the cellular networks containing 5G connectivity have access to mobility of multiple-tier to bring flexibility in mobility management while considering the major factors (Doewes et al., 2021; Ahmed et al., 2021).

For example, the5G cellular networks gets requests from mMTC UEs with components such as immobility, costing and tight energy-efficiency requirements to initiate data transfer reactively (Fig. 1c). Meanwhile, certain vertical businesses have their mobility management tailored by $5 \mathrm{G}$ cellular networks based on geographical features. We can also term it as, once UEs enter a certain region, may provide top level mobility management (e.g., dual connectivity) and thus be able to update their locations more frequently.

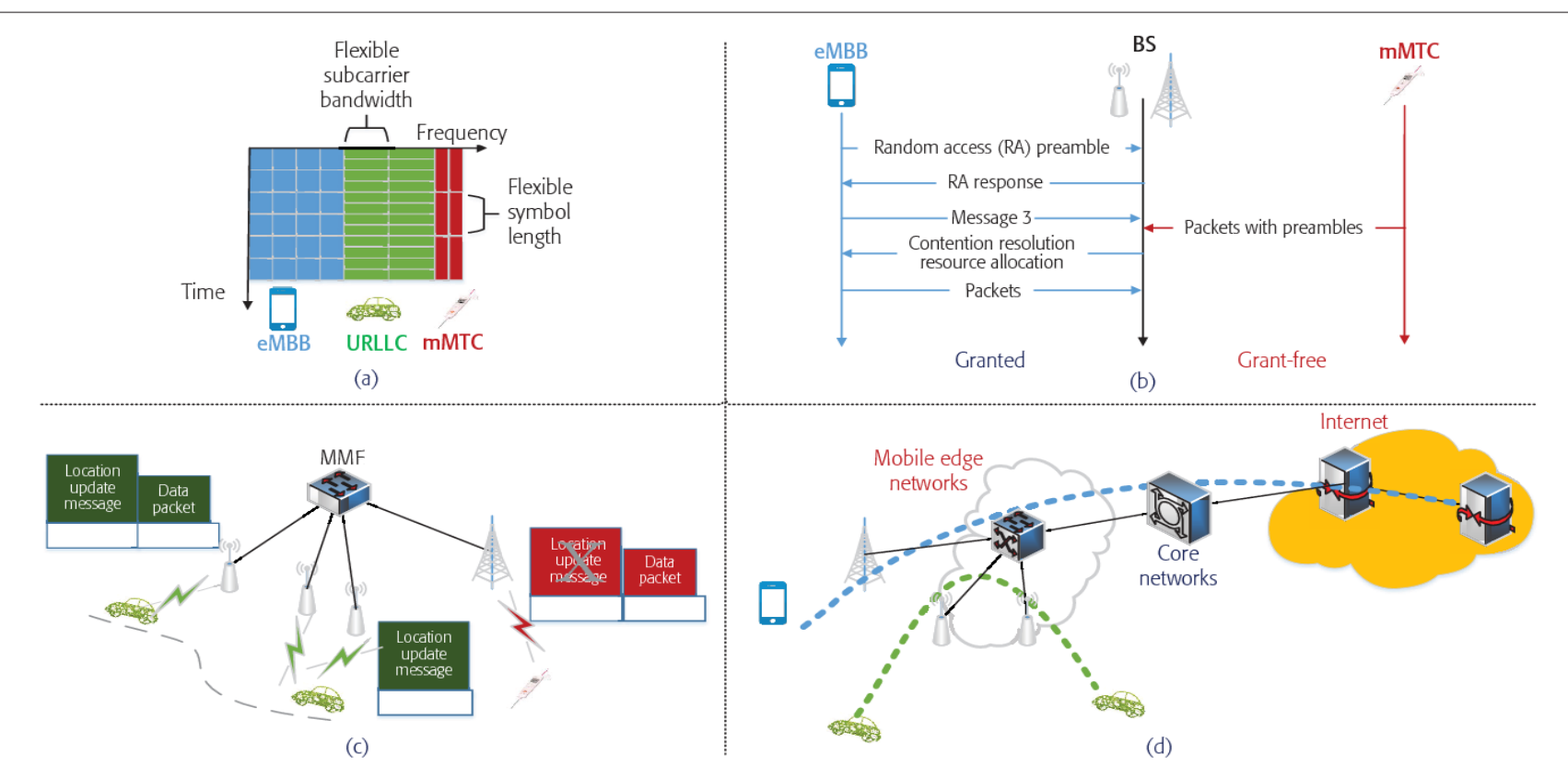

Figure 1: Candidate technologies for intelligent cellular networks: a) flexible bandwidth and symbol length enabled by FBMC and UFMC; b) granted and grant-free transmission enabled by NoMA; c) flexible mobility management schemes; d) dynamic service provisioning stack

\section{Management and Orchestration}

Recently, increasing development of software-defined networks (SDN) has been witnessed by the industry (Li et al., 2014). Several well-known operators and manufacturers, including AT\&T, China Mobile, Telefonica, and Cisco and Huawei, have collaborated to launch the Open Networking Lab, which aims to bring innovation in SDN to the Internet. This is called as Central Office Re-architected is a Data centre (CORD). CORD has effective existing virtual hardware devices including CPE (customer premises equipment), OLT (optical line transmission), and BNG (broadband network gateway) and developed software counterparts includes open network operating system (ONOS) apps on the commodity hardware. Moreover, CORD has its basis at which it offers various software elements which are built in it (Zhou et al., 2016).

\section{Service Provisioning Management}

Besides the interface enabled by FBMC or UFMC, planned by $5 \mathrm{G}$ networks to intelligently forwarding the route of one service using SDN application interfaces (APIs) and have flexibility in service provisioning stack. 5G cellular networks have proposed a novel concept called network slicing as a result of the advancement of SDN and network 
function virtualization (NFV) (NS). NS makes it possible to generate intellectually network pipes to deliver ease in services that demand varying capabilities, performance metrics, and isolation criteria, rather than establishing specialised networks for distinct services. Moreover, it includes anchoring points, and service continuum capabilities. Specifically, mobile edge has potentially evolved to replace its forwarding-only functionality to places the storage capacity, memories, and computational capabilities (Soldani and Manzalini 2020).

As a result, anchoring and forwarding points are chosen by the UEs which include serving gateway in core networks $(\mathrm{CNs})$ and the cellular based network on practical requirements. Services with rigorous requirements for mobility and service continuity, for example, could transfer their initial points to edge networks which provide closer proximity, as shown in Fig. 1(d). In addition, device-to-device (D2D) connectivity in 4G has limited proximity services and public safety messages. However, direct communication with network assistance between vehicles and UEs is becoming a reality, and services of V2X are acquiring major subject to handle better URL. (e.g., automobiles)

5G cellular networks can have an advantage of the advanced yet computation-intensive technologies everywhere from physical layer to the network structure, thanks to Moore's Law's huge advancement in signal processing capabilities. As a result, 5G cellular networks can present other options for many scenarios, which includes intellectuality and meet the requirements for full Artificial Intelligence.

\section{SySTEM MOdeL}

It presents the network model and the spectrum in this section where sensing is performed via a cluster head $(\mathrm{CH})$.

\section{Network Model and Assumption}

A heterogeneous5G network for selection OF spectrum and access is presented. Different primary networks with multiple channel availability are present in the architecture. Each cluster in a primary network contains devices as ' $n$ ' IoT and ' $m$ ' channels, with each cluster consisting of several clusters. We examine a two-fold design in this study, as shown in figure 2, characteristics based on the IoT. The nodes have structured works stored on the devices in situation of the item in IoT relationship between multiple IoT devices.

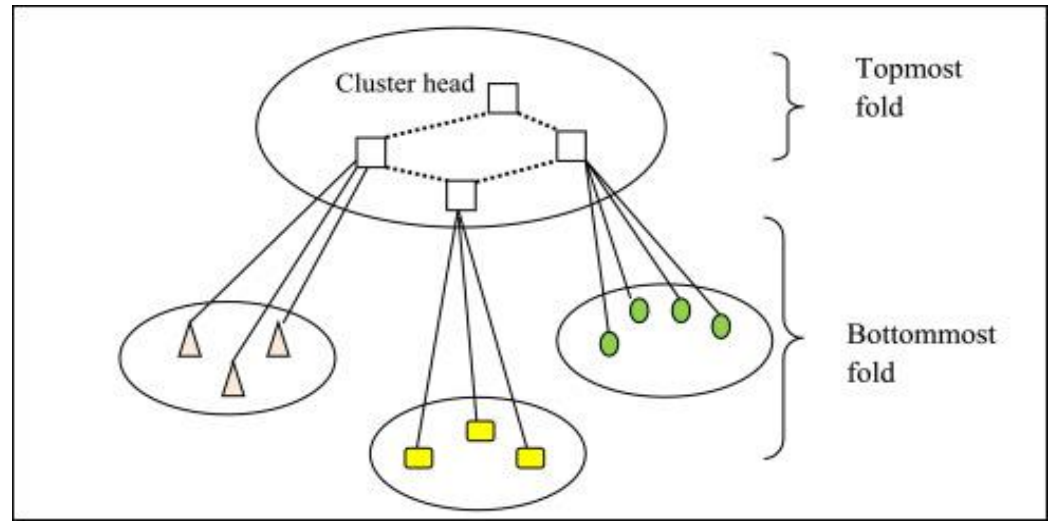

Figure 2: Sample two-fold architecture

In given illustration, the IoT devices are in the section which is a low form multiple mesh networks, and the topology of star forms the topmost networks. In the lowest forms of mesh networks, devices interact with mesh and various figures of $\mathrm{CH}$, and the cluster heads that are linked together.

Shown in the illustration 1 , ' $n$ ' number of devices 'in the lowest fold are indicated as $D=D_{1}, D_{2} \ldots \ldots D_{n}$ ' and with a mesh with device is represented as $D_{1}=D_{11}, D_{12}, D_{13}, \ldots . . D_{1 n}{ }^{\prime}, D_{2}=D_{21}, D_{22}, D_{23}, \ldots \ldots . D_{2 n}$ ' and so on. The $C H$ is represented as $\mathrm{CH}=\mathrm{CH} \mathrm{CH} 2 \ldots \ldots . . . \mathrm{CH}_{n}$ with IoT devices respectively. Considering the spectrum selection techniques which are not registered to IoT devices which is applied via function-based position to check the probability of transmitting to set its position. The specific function is given below,

$F: P_{r} \rightarrow[0,1]$

The function ' $F$ ', and ' $P_{r}$ ' denotes the positional rating, which is indicated as ' $P_{r}=A, I A$, Succ, Fail' in the above equation (1). To acquire access to a spectrum, it must first be in an active $(A)$ or inactive (IA) condition. Here ' $A$ ' is active state which shows spectrum accessibility by the licensed IoT devices. Whichever IoT devices license do not have access to the spectrum, it becomes in an inactive state ' $I A^{\prime}$ ', the spectrum access which is successful is denoted as 'Succ', and spectrum access failure is denoted as 'Fail' respectively. 
Let's say there's no licensed transmission available; but, if IoT device which is unlicensed acquires spectrum access, the likelihood of transmission which is successful for this IoT at the next position is shown below.

$\operatorname{Prob}_{s}(C A)(1-\operatorname{Prob}$ (busy)): $N$

The successful transmission probability 'Probs' is calculated using the possibility of successful accessing the path 'Probs (CA)' and the chances of seeing the channel state as busy 'Prob (busy)' for ' $N$ ' different position numbers using the preceding equation (2). The number of transmissions of IoT device ' $A V_{u}$ ' or the average number of the nonregistered IoT devices for continuous spectrum access is then shown below.

$A V_{u}=1 / 1-\left[\right.$ Prob $\left._{s}\right]$

\section{Energy detection model}

While performing various duties, every IoT device requires a significant limit of energy. The modes which such as inactive mode, active mode, spectrum selection mode, and spectrum access mode all have distinct energy usage. Let ' $P_{S, i}{ }^{\prime}$ be the power utilised by the 'ith' IoT device during the spectrum selection procedure. The total energy used in the spectrum selection procedure is then shown below.

$E_{s s}=P_{S, i} * a_{S}$

The calculation of the energy which is consumed at the procedure of selecting spectrum ' $E_{s s}$ ' consumes power $P_{S, i}$ along with total time taken at the selection of spectrum process 'as' from the previous equation (4). It is recorded that energy is consumed during the procedure of spectrum (i.e. the cluster head). The IoT device needs power ' $\mathrm{P} \mathrm{CH}^{\prime}$ to communicate the head of cluster, it can be explained from the following processes.

$E_{S A}=P_{C H, i} * a_{C H}$

The energy taken in during the procedure of accessing spectrum procedure 'ESA' is calculated using the power which is consumed ' $P C H, I$ and also the time taken at the selection of spectrum process ' $a_{C H}$ ', as shown in equation (5). The total energy used by the 'ith' IoT device is then calculated as follows.

$E C_{i}=E_{S S}+E_{S A}$

The above equation (6), $E C_{i}$ which is denoted by the total energy taken by the 'ith' IoT device. From the above equation (6), $E C_{i}$ indicates the consumption of total energy ' $i t h$ ' IoT device.

\section{Fractional knapsack and langrange hyperplane spectrum access network}

IoT devices create large amounts of data. The rapid development of the term IoT is constrained by finite spectrum of radio. This is owing to the fact that IoT networks use a different frequency than other networks. The spectrum, on the other hand, is not particularly rare in frequency, but it is claimed to be underutilised, and it is presently selected by suitably applying radio environment information. FK-LHSA framework is used for network of IoT sensor is developed in this paper to improve spectrum access with complete accuracy while minimising spectrum access latency. The FK-LHSA framework is shown in Figure 3.

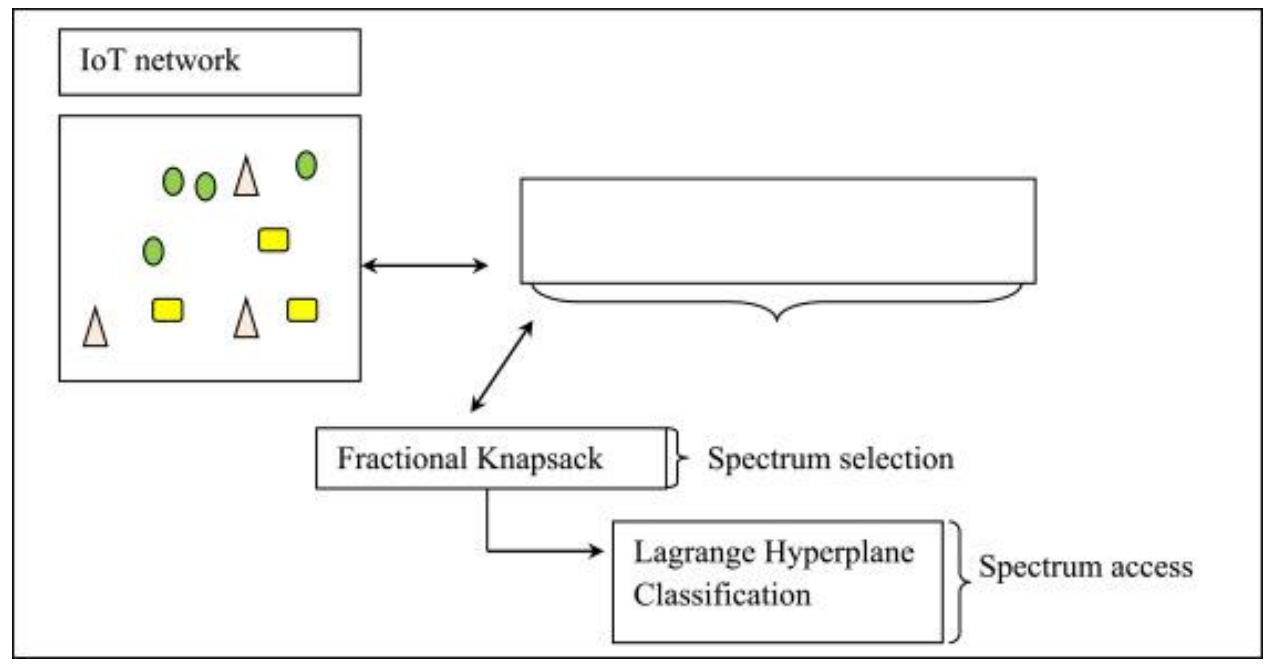

Figure 3: Block diagram of FK-LHSA framework 
The division of framework of FK-LHSA takes place into two different categories, as shown in the illustration above. In the beginning, the EPSDLAC Technique gets input from an IoT network. The early processing is used to filter out irrelevant data and focus on what's important. To lower the time complexity and dimensionality, pre-processing is done. The Fractional Knapsack model is used to pick the spectrum in the first stage. Spectrum access is accomplished in the second stage using Lagrange Hyperplane Classification. The following sections provide a full description of the framework mentioned below.

\section{Fractional knapsack multiband spectrum selection}

The Non-Orthogonal Multiple Access (NOMA) has gotten a lot of attention in recent years and achieved necessary technology for 5G networks (Wang et al., 2020). It has various advantages over the conventional multiple access approach, which allows multiple users to share a single resource block while taking advantage of differences channel between various devices of IoT. Using the NOMA and Fractional Knapsack energy, the suggested model ensures that each IoT device has the ideal rate of data requirement and consumption of energy. To begin, each IoT device determines whether or not to participate in the multi-band spectrum selection process based on if the residual energy accessibility. The $\mathrm{CH}$ then becomes certain about the spectrum to Iot devices that have great amount of energy to perform multiband spectrum selection, whereas low-energy IoT devices do nothing till the end of the position selection process. The $\mathrm{CH}$ then counts the result of selected spectrum from IoT devices and runs a series of tests to evaluate whether primary users exist on each frequency.

A FKMSS model is used in this study. When it is proposed to few things, such as IoT devices and channels, data is specified at a minimum rate during the Spectrum Selection process if it has enough energy and data to broadcast. $\mathrm{CH}$ now has complete grip on all ' $m$ ' channels; it may schedule the spectrum to all the devices of IoT based on the IoT devices requirements. A collection of subset (i.e. whether the selected path for an IoT device or not) it is identified using this Fractional Knapsack, therefore total number of channels which is to be sorted is less than or equal to a threshold given the complete value should be as large as possible. With numerous IoT devices evaluated in this study, each set of IoT devices is broken down into smaller portions (i.e. fractions), resulting in the FKMSS model. Figure 4 depicts the planned flow diagram.

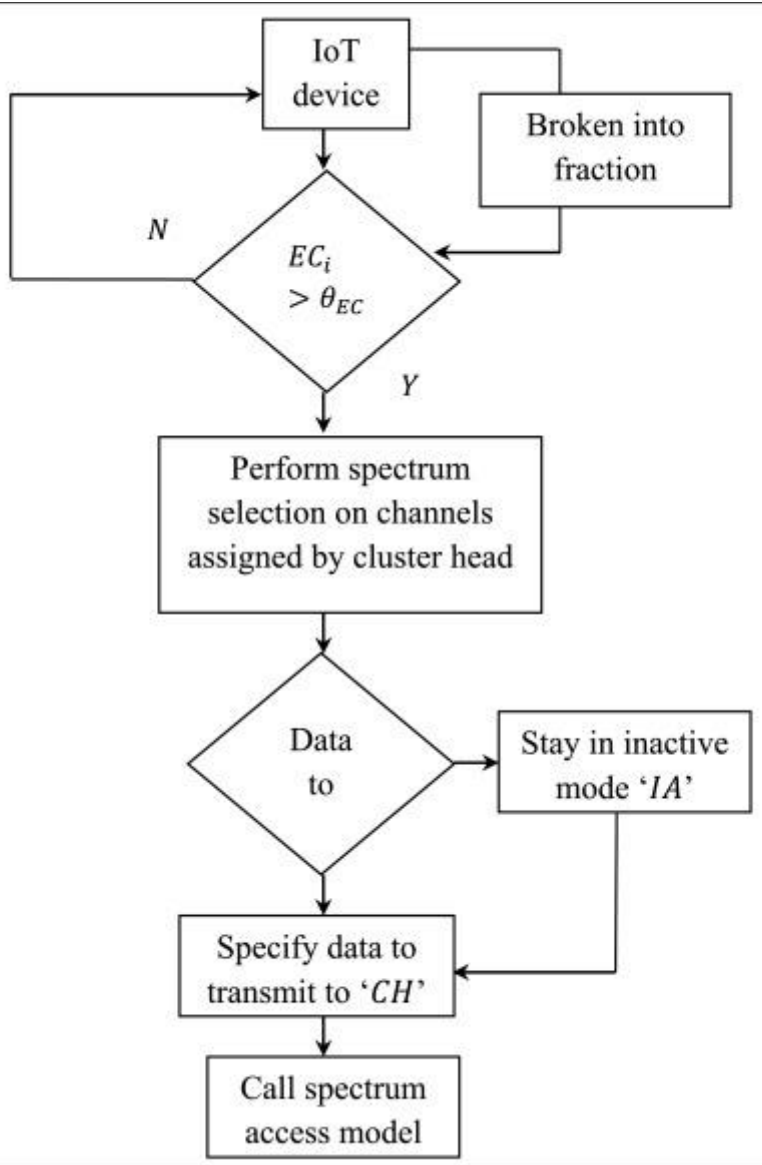

Figure 4: Flow diagram of FKMSS model 
The goal of the FKMSS model's architecture, as indicated in the diagram above, is to make ways in a way that overall throughout is maximised. As the data transmission and energy emitted (i.e. set of candidates) for the same IoT device on the 'ith' channel are not claimed to be at same duration, a dual index outcome ' $D$ 'is given as follows. $D_{j i}=\{1$, if ith user is using ith channel 0$\}$

The best candidate is found using the resultant equation obtained from (7). Following that, a plausible solution is found using a dual index. The data transmission or energy consumption of each IoT then the node is scheduled. The scheduling of the ' $j t h$ ' IoT node is denoted by the dual index ' $j$,' where "when the 'jth' node is scheduled for data transmission" and "when the 'jth' node is scheduled for energy consumption." It is stated numerically as follows. $\xi j=\{1$ if ith IoT node transmit data

\section{0, if ith IoT node measures energy\}}

The ultimate goal of the model of RMSS utilising greedy technique, as shown in equations (7) and (8), is to improve channel throughput while optimising ' $D_{j i}$ ' and ' $\xi j$ ' for all $i$ and ' $j$ '. The pseudo-code given below is the depiction of Dual Index Spectrum Selection.

The technology of IoT ' $j$ ', channels $i$ and ' $\mathrm{CH}$ ' are among first possibilities to be activated. The selection function is the following stage, which chooses the best candidate (based on usage of energy) for the purpose of communication and selection of spectrum with cluster head. The other stage is to determine the IoT device and channel can be utilised to design a solution utilising a dual index outcome using a feasible solution. Finally, a function is measured to verify the way out utterly (i.e., transmission of data or energy measurement).

\section{Langrange hyperplane optimum spectrum access}

In the advanced years of computer and technology the working of machines and AI have conquered the interest of academics and researches done by people brought establishment in wireless communication, with the goal of using AI for 5G (Bynagari, 2015; Manavalan, 2019b). By various procedures such as breaking down, accessibility and spectrum allocation, an $\mathrm{AI}$ is also known for $5 \mathrm{G}$ network which gives cluster leaders the ability to establish a proper data store (Song et al., 2020).

When the spectrum is not distributed appropriately, however, its occurrence is to have during the transmission from device-to-device. In order to reduce interference, an $\mathrm{x}$ model is employed in this study to allocate spectrum in a fair and optimal manner. Vector Machine is also used to guide that solve sand classifies the problem by increasing the margin while reducing the total problems in this paper. Consider the following utility function for each IoT user. $U_{i}=s_{i} S B\left(a_{i}\right)$, where $S B=S B_{1}+S B_{2}+\cdots+S B_{n}$

The utility ' $U_{i}$ ' for each IoT user, ' $i$ ' is calculated using the service type parameter $s_{i}$, the needed spectrum band 'SB' and the SINR value ' $a$ ' from the previous equation (9). Even when IoT devices have the same spectrum bandwidth, the parameter of service relates to the service which transmits are the kinds that are determined to be distinct. $a_{i}=\frac{p l_{i i}}{p l_{i j}+N}$

The receiver which is at SINR 'ai' is calculated using when the route is lost during the IoT transition 'plii' and the IoT receiver $p l_{i j}{ }^{\prime}$, as well as an additional factor of noise ' $N$ ' as shown in the above equation (10). Furthermore, the genuine gains of IoT devices are examined from the standpoint of capacity. Assuming that all IoT devices use the approved channels, which are limited by their power, also depends upon the capacity of spectrum transmitted; the sub-channel is represented as follows.

$T C_{j}=\sum_{i, j=1}^{n} T_{i j} \operatorname{Exp}_{p l 1 i} \operatorname{Exp}_{p l 0 i} *\left[S B\left(\frac{p l_{1 i} D_{i j}}{S B}\right)\right]$

The capacity at which transmission takes place ' $T C^{\prime}$ ' of the user of IoT or device ' $J$ ' is calculated using the ' $T$ ' time of an idle channel ' $i$ ' path that is lost from an IoT user 'Exppli' ', and loss of path to an IoT user 'Expploi' ", spectrum band ' $S B^{\prime}$ respectively, from the preceding equation (11). The following equation is believed to be obtained optimal spectrum access by solving problem is known as Lagrange function. $L\left(D_{j}, \beta\right)=\sum_{i, j=1}^{n} T_{i j} \operatorname{Exp}_{p l 1 i} \operatorname{Exp}_{p l 0 i} *\left[S B\left(\frac{p l_{1 i} D_{i j}}{S B}\right)\right]-\sum_{i=1}^{n} \beta_{i}\left(\operatorname{Exp}_{p l 1 i} \operatorname{Exp}_{p l 0 i}\left(D_{i j}\right)-D_{0 i}\right)$

$\sum_{i=1}^{n} \operatorname{Exp}_{p l 1 i} \operatorname{Exp}_{p l 0 i}\left[T_{i j} S B\left(\frac{p l_{1 i} D_{i j}}{S B}\right)\right]-\beta_{i}\left(D_{i j}-D_{0 i}\right)$

The optimal access of the spectrum for an IoT device ' $D_{j}$ ', is done by using the above equations (12) and (13) and a Lagrange factor ' $\beta$ '. Finally, looking at the equation Lagrange function above it yields maximum capacity of spectrum access, which can be shown as follows. 
$D_{i j}^{\prime}=\left\{\begin{array}{c}\left.\text { if } p l_{i} \geq \frac{N}{T_{i j}}, w * D_{i j}^{\prime}-b=1, \text { [optimal sSpectrum allocation }\right] \\ \text { if } p l_{1 i}<\frac{N}{T_{i j}}, w * D_{i j}^{\prime}-b=-1[\text { No optimally arrived }] \\ \left.w * D_{i j}^{\prime}-b=0, \text { Otherwise [idle state }\right]\end{array}\right\}$

The goal of our work using the resulting Lagrange function is to separate the two classes from the available examples. As stated above (14) the classifier must meet the following requirements, with ' $w$ 'denotes weight and ' $b$ ' indicating bias value. The LH Classification model is depicted in the diagram below.

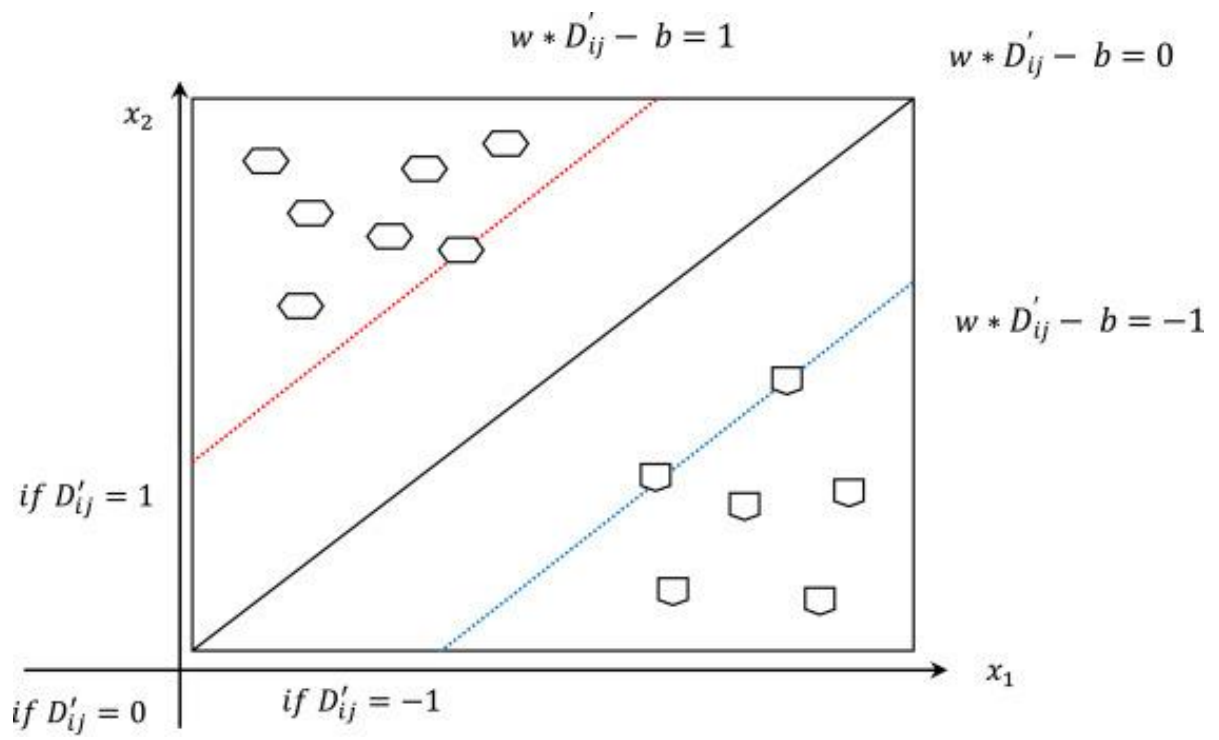

Figure 5: Hyperplane classifying spectrum into two different classes

In the given equation above, the classification of spectrum can be treated in two different ways which can be used to increase the margin with least possibilities of errors in it. The two categories could the optimal or the non- optimal spectrum. When the division of spectrum is carried out in a positive manner, we receive optimal spectrum results.

From the LH access to spectrum technique mentioned above, four phases are taken to optimize accessibility to the spectrum with least latency to spectrum access and to increase accuracy of spectrum. The function for the entire utility IoT device is obtained in the first stage. The second stage examines the capacity at which transmission take place on a different channel after that. The final phase entails using the function of Lagrange to achieve maximum results of spectrum accessibility. Finally, the classification results are obtained using the derivative of the Lagrange function.

\section{CONCLUSION}

In this paper, we propose that AI be used to cellular networks. The intelligence required in developing practically in all areasof cellular networks operating 5G, includes managements such asthe radio resource management, the general management, mobility management and orchestration, and provisioning management of services, were first one to be explored. Second, we suggested an AI which has access to greedy IoT framework for selection of selection and spectrum accessibility. In the licenced band, free spectral channels were discovered and assigned to the appropriate IoT devices. A CH makes the choice on how these channels are assigned. The spectrum is then chosen using the NOMA and Fractional Knapsack energy models. The validity of the suggested framework is demonstrated by the results for various parameters under various models. As a result, future study can focus on analysing complex timings by using various techniques for learning machine operation. To increase spectrum performance, the dataanalytics-of AI based spectrum allocation (ADASA) algorithm is applied.

\section{REFERENCES}

Ahmed, A. A. A., \& Ganapathy, A. (2021). Creation of Automated Content with Embedded Artificial Intelligence: A Study on Learning Management System for Educational Entrepreneurship. Academy of Entrepreneurship Journal, 27(3), 1-10, https://doi.org/10.5281/zenodo.4973057

Ahmed, A. A. A., Aljarbouh, A., Donepudi, P. K., \& Choi, M. S. (2021). Detecting Fake News using Machine Learning: A Systematic Literature Review. Psychology and Education, 58(1), 1932-1939. https://doi.org/10.17762/pae.v58i1.1046 
Amin, R., \& Manavalan, M. (2017). Modeling Long Short-Term Memory in Quantum Optical Experiments. International Journal of Reciprocal Symmetry and Physical Sciences, 4, 6-13. Retrieved from https://upright.pub/index.php/ijrsps/article/view/48

Azmat, F., Chen, Y., Stocks, N. (2016). Analysis of Spectrum Occupancy Using Machine Learning Algorithms. IEEE Transactions on Vehicular Technology, 65(9), 6853 - 6860. https://doi.org/10.1109/TVT.2015.2487047

Bynagari, N. B. \& Ahmed, A. A. A. (2021). Anti-Money Laundering Recognition through the Gradient Boosting Classifier. Academy of Accounting and Financial Studies Journal, 25(5), 1-11. https://doi.org/10.5281/zenodo.5523918

Bynagari, N. B. (2015). Machine Learning and Artificial Intelligence in Online Fake Transaction Alerting. Engineering International, 3(2), 115-126. https://doi.org/10.18034/ei.v3i2.566

Bynagari, N. B. (2019). GANs Trained by a Two Time-Scale Update Rule Converge to a Local Nash Equilibrium. Asian Journal of Applied Science and Engineering, 8, 25-34. Retrieved from https://upright.pub/index.php/ajase/article/view/32

Bynagari, N. B., \& Amin, R. (2019). Information Acquisition Driven by Reinforcement in Non-Deterministic Environments. American Journal of Trade and Policy, 6(3), 107-112. https://doi.org/10.18034/ajtp.v6i3.569

Doewes, R. I.; Ahmed, A. A. A.; Bhagat, A.; Nair, R.; Donepudi, P. K.; Goon, S.; Jain, V.; Gupta, S.; Rathore, N. K.; Jain, N. K. (2021). A regression analysis based system for sentiment analysis and a method thereof. Australian Official Journal of Patents, 35(17), Patent number: 2021101792. https://lnkd.in/gwsbbXa

Fadziso, T., \& Manavalan, M. (2017). Identical by Descent (IBD): Investigation of the Genetic Ties between Africans, Denisovans, and Neandertals. Asian Journal of Humanity, Art and Literature, 4(2), 157-170. https://doi.org/10.18034/ajhal.v4i2.582

Fan, C., Li, B., Zhao, C., Guo W. and Liang, YC. (2018). Learning-Based Spectrum Sharing and Spatial Reuse in mm-Wave Ultradense Networks. IEEE Transactions on Vehicular Technology, 67(6), 4954-4968. https://doi.org/10.1109/TVT.2017.2750801

Huang, Y., Tan, J. \& Liang, YC. (2017). Wireless big data: transforming heterogeneous networks to smart networks. Journal of Communications and Information Networks. 2, 19-32. https://doi.org/10.1007/s41650-017-0002-1

I, CL., Han, S., Xu, Z., Wang, S., Sun, Q., Chen, Y. (2016). New Paradigm of 5G Wireless Internet. IEEE Journal on Selected Areas in Communications, 34(3), 474-482. https://doi.org/10.1109/JSAC.2016.2525739

Li, R., Zhao, Z., Zhou, X., Palicot, J. and Zhang, H. (2014). The prediction analysis of cellular radio access network traffic: From entropy theory to networking practice. IEEE Communications Magazine, 52(6), $234-240$. https://doi.org/10.1109/MCOM.2014.6829969

Li, Z. and Guo, C. (2020). Multi-Agent Deep Reinforcement Learning Based Spectrum Allocation for D2D Underlay Communications. IEEE Transactions on Vehicular Technology, 69(2), 1828-1840. https://doi.org/10.1109/TVT.2019.2961405

Li, Z. and Guo, C. (2019). Multi-Agent Deep Reinforcement Learning based Spectrum Allocation for D2D Underlay Communications. Networking and Internet Architecture. https://arxiv.org/abs/1912.09302

Lin, K., Li, C., Tian, D., Ghoneim, A., Hossain, M. S., Amin, S. U. (2019). Artificial-Intelligence-Based Data Analytics for Cognitive Communication in Heterogeneous Wireless Networks. IEEE Wireless Communications, $26(3), 83-89$. https://doi.org/10.1109/MWC.2019.1800351

Manavalan, M. (2019a). P-SVM Gene Selection for Automated Microarray Categorization. International Journal of Reciprocal Symmetry and Physical Sciences, 6, 1-7. Retrieved from https://upright.pub/index.php/ijrsps/article/view/43

Manavalan, M. (2019b). Using Fuzzy Equivalence Relations to Model Position Specificity in Sequence Kernels. Asian Journal of Applied Science and Engineering, 8, 51-64. Retrieved from https://upright.pub/index.php/ajase/article/view/42

Manavalan, M. (2020). Intersection of Artificial Intelligence, Machine Learning, and Internet of Things - An Economic Overview. Global Disclosure of Economics and Business, 9(2), 119-128. https://doi.org/10.18034/gdeb.v9i2.584

Manavalan, M., \& Chisty, N. M. A. (2019). Visualizing the Impact of Cyberattacks on Web-Based Transactions on LargeScale Data and Knowledge-Based Systems. Engineering International, 7(2), 95-104. https://doi.org/10.18034/ei.v7i2.578

Sharma, D. K., Chakravarthi, D. S., Shaikh, A. A., Ahmed, A. A. A., Jaiswal, S., Naved, M. (2021). The aspect of vast data management problem in healthcare sector and implementation of cloud computing technique. Materials Today: Proceedings. https://doi.org/10.1016/j.matpr.2021.07.388 
Soldani, D. and Manzalini, A. (2015). Horizon 2020 and Beyond: On the 5G Operating System for a True Digital Society. IEEE Vehic. Tech. Mag., 10(1), 32-42.

Song, H., Bai, J., Yi, Y., Wu, J. and Liu, L. (2020). Artificial Intelligence Enabled Internet of Things: Network Architecture and Spectrum Access. IEEE Computational Intelligence Magazine, 15(1), 44-51. https://doi.org/10.1109/MCI.2019.2954643

Wang, J., Jiang, C., Zhang, H., Ren, Y., Chen, KC. and Hanzo, L. (2020). Thirty Years of Machine Learning: The Road to Pareto-Optimal Wireless Networks. IEEE Communications Surveys $\mathcal{E}$ Tutorials, 22(3), 1472-1514. https://doi.org/10.1109/COMST.2020.2965856

Wang, X., Li, X., and Leung, V. C. M. (2015). Artificial Intelligence-Based Techniques for Emerging Heterogeneous Network: State of the Arts, Opportunities, and Challenges. IEEE Access, 3, 1379-1391.

Yao, M., Sohul, M., Marojevic, V. and Reed, J. H. (2019). Artificial Intelligence Defined 5G Radio Access Networks. IEEE Communications Magazine, 57(3), 14-20. https://doi.org/10.1109/MCOM.2019.1800629

Zhou, X., Li, R., Chen, T., Zhang, H. (2016). Network slicing as a service: enabling enterprises' own software-defined cellular networks. IEEE Communications Magazine, 54(7), 146-153. https://doi.org/10.1109/MCOM.2016.7509393

$--0--$ 